\title{
Failing Vector Identification Based on Overlapping Intervals of Test Vectors in a Scan-BIST Environment
}

\author{
Chunsheng Liu, Student Member, IEEE, and Krishnendu Chakrabarty, Senior Member, IEEE
}

\begin{abstract}
We present a new scan built-in self-test (BIST) approach for determining failing vectors for fault diagnosis. This approach is based on the application of overlapping intervals of test vectors to the circuit under test, and it is especially suitable for faults that are detected by a relatively small number of pseudorandom test patterns. Two multiple-input signature registers are used in an interleaved fashion to generate intermediate signatures, thereby obviating the need for multiple test sessions. The knowledge of failing and fault-free intervals is used to obtain a set $S$ of candidate failing vectors that includes all the actual (true) failing vectors. We propose a signature-analysis method based on overlapping sections and the principle of superposition to effectively prune the candidate set. We present analytical results to determine an appropriate interval length and the degree of overlap, as well as upper and lower bounds on the size of $S$. We also determine a lower bound on the number of true failing vectors through a simple graph model. Finally, we present experimental results for the ISCAS'89 benchmark circuits to demonstrate the effectiveness of the proposed scan-BIST diagnosis approach.
\end{abstract}

Index Terms-Candidate vector set, diagnostic resolution, fault diagnosis, signature analysis, superposition.

\section{INTRODUCTION}

A S PROCESS technologies shrink and designs become more complex, built-in self-test (BIST) is gaining increasing acceptance as an industry-wide test solution [1]. In particular, the combination of scan design and BIST, commonly referred to as scan-BIST, is now especially common [2]. Scan-BIST techniques typically apply a large number of patterns from a pseudorandom pattern generator (PRPG) to the circuit under test (CUT) via scan chains. The test responses are then captured by the scan chain and a compact signature is generated using a multiple-input signature register (MISR); see Fig. 1. However, a problem with this approach is that the signature provided by the MISR does not contain enough diagnostic information, either to identify failing vectors or to precisely identify error-capturing scan cells. The pass/fail information obtained from the MISR at the end of the test session is usually insufficient to diagnose the failure via effect-cause analysis.

Fault diagnosis is essential for the identification of manufacturing defects and for yield learning. The cost of diagnosis is proportional to the time required for failure analysis, which can be extremely high for a scan-BIST scheme involving

Manuscript received May 24, 2002; revised September 26, 2002. This work was supported in part by the National Science Foundation under Grant CCR9875324 and Grant CCR-0204077. This paper was recommended by Associate Editor N. K. Jha.

The authors are with the Department of Electrical and Computer Engineering, Duke University, Durham, NC 27708 USA (e-mail: krish@ee.duke.edu).

Digital Object Identifier 10.1109/TCAD.2003.810739

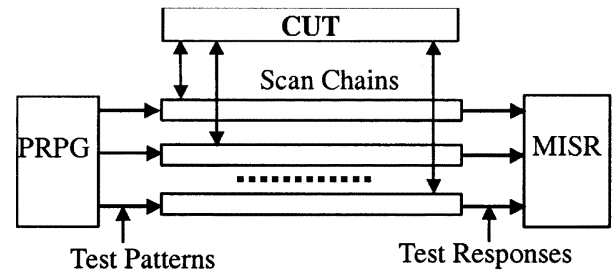

Fig. 1. Generic scan-BIST scheme.

tens of thousands or millions of vectors [3]. Therefore, there is a pressing need for BIST schemes that provide adequate diagnostic information, without burdening the failure analysis process with superfluous information.

The diagnostic information in scan-BIST can be classified as space information and time information, respectively. The former refers to the set of scan cells that capture errors during the BIST session. This problem has received a lot of attention recently, and a number of methods involving scan-chain partitioning with multiple test sessions have been proposed for precisely identifying the failing scan cells [4]-[7]. A more difficult problem in scan-BIST diagnosis is that of identifying the set of failing vectors. This is because the length of a scan chain in a typical BIST scheme is usually much smaller than the number of test vectors applied to the CUT. As a result, fewer practical techniques are available today for rapidly identifying a small set of candidate failing vectors.

Early work on failing vector identification was based on the analysis of linear-feedback shift-register sequences [8], the use of cycling registers [9], and error-correcting codes [10], [11]. An alternative approach that does not require intermediate signatures was presented in [12]. These techniques suffer from the drawback of limitations on error multiplicity [8], diagnostic aliasing [9]-[11], and high overhead [10], [12]. Recently, a method based on the combination of cycling registers and pruning techniques was proposed for failing vector identification [13]. While this approach is useful in narrowing down the set of candidate failing vectors, it suffers from the drawback that it does not identify all the failing vectors.

In this paper, we present a technique for failing vector identification based on the use of overlapping intervals of test vectors and the principle of superposition. This approach is especially suitable for diagnosing faults that are detected by a small number of pseudorandom patterns, i.e., have relatively low detection probability. An interval is a set of consecutive test vectors. An advantage of this approach is that all failing vectors are included in a reduced set of candidate vectors for failure analysis. Unlike the approach based on test windows [18], where test vectors are partitioned into nonoverlapping segments, our 
method is based on overlapping intervals of test vectors. In combination with a novel pruning procedure, the overlap allows us to prune the set of candidate failing vectors more efficiently. An interval that does not contain a failing vector can be omitted from the set of candidate failing vectors. The overlap ensures that if a failing interval $I_{1}$ is followed by a nonfailing interval $I_{2}$, only the set difference $I_{1}-I_{2}$ needs to be retained in the set of candidate failing vectors. As an enhancement to the basic interval method proposed in [19], we show here that interval overlap also allows us to use a simple yet efficient signature analysis technique based on superposition that can significantly prune the candidate set. This pruning method does not require any additional information. In order to reduce the candidate set further, postprocessing procedures can be performed as optional steps [19], which require additional signatures.

The proposed interval-based approach also allows us to determine upper and lower bounds on the number of candidate failing vectors, as well as a lower bound on the number of actual (true) failing vectors. The lower bound on the number of true failing vectors is obtained via a simple graph model based on the pass/fail status of the intervals.

In addition to facilitating effect-cause analysis, the proposed approach can also benefit cause-effect analysis [14]. For example, the knowledge of candidate failing vectors can improve the resolution provided by a compact fault dictionary [15].

The organization of this paper is as follows. In Section II, we present the scan-BIST architecture for the proposed intervalbased scheme. In Section III, we present an analytical approach to determine the interval length and the amount of overlap. We also determine upper and lower bounds on the number of candidate failing vectors and a lower bound on the number of true failing vectors. In Section IV, we present a simple signatureanalysis technique where overlap is used to reduce the candidate set without additional signature information. We then present updated upper and lower bounds on the size of the candidate set when this pruning step is used. Finally in Section V, we present experimental results for the larger ISCAS' 89 benchmark circuits.

\section{INTERVAL-BASED DiAGNOSIS}

In this section, we introduce the notion of intervals of test vectors for diagnosis. Let $T$ be an ordered set of patterns that is applied to the CUT by the test pattern generator (TPG). An interval $I$ corresponds to a subset of consecutive vectors from $T$. The basic idea of interval-based diagnosis is to divide $T$ into a set of overlapping intervals $I_{1}, I_{2}, \ldots, I_{N}$ such that $T=I_{1} \cup$ $I_{2} \cup \cdots \cup I_{N}$ and $I_{i} \cap I_{i-1} \neq \Phi$.

Fig. 2 illustrates three consecutive intervals. The entire test sequence is split into intervals of length $L$ and overlap $r$. Note that $I_{i}$ represents the $i$ th interval. The test patterns in an interval are applied to the CUT and the signature is compared for every interval. We assume that the aliasing in the MISR used for signature analysis can be neglected. If an interval contains one or more failing vectors, the corresponding signature is different from the fault-free signature. The diagnosis procedure, therefore, relies on the knowledge of failing intervals, from which the candidate failing vectors are derived.

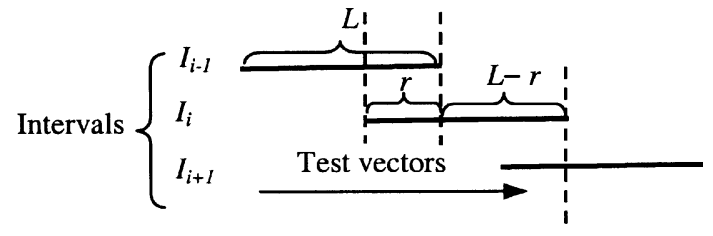

Fig. 2. Test patterns in intervals.

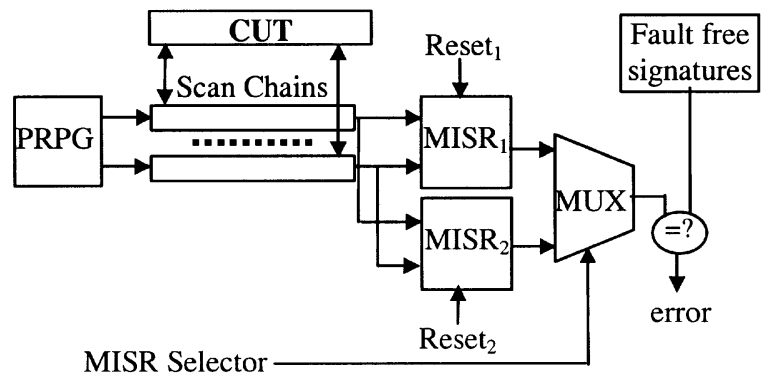

Fig. 3. Scan-BIST architecture for interval-based diagnosis.

A hardware implementation of the proposed scheme is shown in Fig. 3. We augment the basic scan-BIST scheme in Fig. 1 by using two MISRs with reset input to capture the signature for every interval. The "MISR Selector" determines the start and stop positions of the intervals and, thus, controls the multiplexer to output the signature from one MISR at a time. Since two adjacent intervals are overlapping, two output MISRs are used to avoid applying the vectors twice. If MISR ${ }_{1}$ is used for the current interval, then $\mathrm{MISR}_{2}$ is selected at the start of the next interval. Therefore, MISR 1 captures signature of the current interval while $\mathrm{MISR}_{2}$ captures signature of the next, and these MISRs provide the interval signatures in an interleaved fashion. The fault-free signatures can be stored off-chip since the intermediate signatures can be downloaded after every interval. An alternative approach is to use time redundancy and a standard BIST scheme based on a single MISR. Note that the proposed interval-based approach may potentially be combined with signature analysis methods that use multiple intermediate signatures but a single reference signature [17]. This offers the advantage of a reduced storage requirement for diagnosis - the details are currently being investigated.

The use of overlapping intervals allows us to reduce the number of candidate failing vectors. This can be explained as follows: If $I_{i}$ is a failing interval and $I_{i-1}$ is not a failing interval, the set of vectors in $I_{i} \cap I_{i-1}$ can be eliminated from the set of candidate failing vectors. In this way, $I_{i}$ and $I_{i-1}$ together contribute only $L-r$ vectors to the set of candidate failing vectors. In order to limit the number of intervals and MISR signatures, we assume that a vector belongs to at most two intervals. In general, let $I_{\mathrm{F}}$ be the set of failing intervals and let $I_{\mathrm{NF}}$ be the set of nonfailing intervals. The set of candidate failing vectors $S$ is then given by

$$
S=\bigcup_{i \in I_{\mathrm{F}}} I_{i}-\bigcup_{j \in I_{\mathrm{NF}}} I j .
$$

An appropriate value of interval length $L$ must be chosen to trade off failing vector resolution with the test application time 


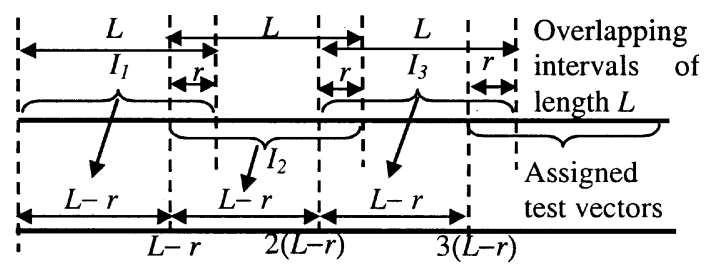

Fig. 4. Test vector assignment for calculating size of $S$.

and storage cost. Short intervals lead to better failing vector resolution; however, they require a larger number of signatures for diagnosis. We determine $L$ using a preprocessing step that estimates the fault detection probability $\alpha$. The preprocessing step is motivated by the fact that the use of intervals is more effective when every interval contains on average less than one failing vector. The preprocessing for interval-based diagnosis is performed by a sampling technique. We apply a small number of vectors $N_{i}$ (typically two to three orders of magnitude less than the total number of BIST vectors $N$ ) to the faulty CUT. We set the interval length $L$ to one and determine the number of failing vectors $D$. (Faults in the CUT might lead to one or more failing vectors.) The detection probability $\alpha$ is estimated as $D / N_{i}$ and $L$ is estimated by $\lfloor 1 / 2 \alpha\rfloor$, which is half the average distance between two failing vectors. This estimate increases the likelihood that failing intervals are separated by nonfailing intervals, which leads to smaller $S$. Note that $\lfloor 1 / 2 \alpha\rfloor$ represents a threshold on $L$; for larger value of $L$, the resolution is usually unsatisfactory. In practice, we should choose a smaller value of $L$ to obtain higher resolution. Since we are considering hard-to-detect faults, it is possible that all the $N_{i}$ samples are fault free. In this case, we use a predefined small interval length $L=L_{0}$, which can be determined by balancing the specific requirements on diagnostic resolution and storage capacity of signatures. Experiment results in Section V show that the use of a small value of $L_{0}$ does not lead to unreasonable increase on the number of signatures and, therefore, diagnostic time.

\section{AnALytical Results}

In this section, we analyze the effectiveness of interval-based diagnosis. We characterize the failing vector candidate set $S$ in terms of $L$ and $r$. To ensure that we do not include in our analysis test vectors that belong to two overlapping intervals twice, we assign the first $L-r$ vectors $(1, \ldots, L-r)$ to the first interval, the second $L-r$ vectors $(L-r+1, \ldots, 2(L-r))$ to the second interval, and so on. This is illustrated in Fig. 4. Let $\alpha$ be the probability that a faulty CUT is detected by a vector from the BIST test set. The probability $p$ that an interval is failing is given by

$$
p=1-(1-\alpha)^{L} .
$$

Consider now two consecutive intervals $I_{i-1}, I_{i}$. The function $v\left(I_{i}\right)$ is used to denote the failing/nonfailing status of the interval $I_{i}$. Table I lists the four possible combinations of the status (failing/nonfailing) of the intervals. Note that a zero in the first two columns of the table indicates that the interval is nonfailing, and a one indicates that the interval is failing. The rightmost column gives the contribution of $I_{i}$ to the set $S$ corresponding
TABLE I

CONTRIBUTION OF INTERVALS TO THE FAILING VECTOR SET

\begin{tabular}{c|c|c|c}
\hline $\boldsymbol{v}\left(\boldsymbol{I}_{i-1}\right)$ & $\boldsymbol{v}\left(\boldsymbol{I}_{i}\right)$ & $\begin{array}{c}\text { Probability of } \\
\text { occurrence }\end{array}$ & Contribution to S \\
\hline 0 & 0 & $(I-p)\left(I-p_{I}\right)$ & 0 \\
\hline 0 & 1 & $(1-p) p_{I}$ & $L-2 r$ \\
\hline 1 & 0 & $p_{I}(I-p)$ & 0 \\
\hline 1 & 1 & $p-p_{I}+p p_{I}$ & $L-r$ \\
\hline
\end{tabular}

to each combination. For example, if $v\left(I_{i-1}\right) v\left(I_{i}\right)=01$, the contribution is $L-2 r$; if $v\left(I_{i-1}\right) v\left(I_{i}\right)=11$, the contribution is $L-r$. The total contribution of the combinations due to $I_{i}$ can be obtained by simply adding the entries in the last column. However, each entry must be weighted with the corresponding probability measure. We introduce two additional parameters $p_{1}\left(p_{2}\right)$, which denote the probabilities that the nonoverlapping (overlapping) section is failing

$$
\begin{aligned}
& p_{1}=1-(1-\alpha)^{L-r} \\
& p_{2}=1-(1-\alpha)^{r} .
\end{aligned}
$$

While the parameter $p_{1}$ is used here, the parameter $p_{2}$ is used in Section IV. If $N$ is the number of BIST test vectors, the number of intervals $k$ is given by

$$
k=\left\lceil\frac{N}{L-r}\right\rceil .
$$

The weighted sum leads to the following expression of $|S|$, the size of the candidate failing vector set:

$$
\begin{aligned}
|S| & =k\left[(1-p) p_{1}(L-2 r)+\left(p-p_{1}+p p_{1}\right)(L-r)\right] \\
& =k\left(p L-p r-p_{1} r+p p_{1} r\right) .
\end{aligned}
$$

Note that here we ignore the first and the last interval. These boundary cases can be omitted because the BIST length $N$ is much greater than the interval length $L$. Since $1>p>p_{1}$, and for the sake of efficiency we assume that a vector can belong to at most two intervals (i.e., $r \leq L / 2$ ), (1) shows that $|S|$ decreases as $r$ is increased from 1 to $L / 2$. In the extreme case of $r=L / 2,|S|=N\left(p-p_{1}+p p_{1}\right)$.

Fig. 5 plots the size of $S$ as a function of $L$ for $r=L / 3$, $N=10000$ and various values of $\alpha$. As expected, we find that for a given circuit characterized by a certain value of $\alpha$, using smaller interval length $L$ results in smaller $|S|$, which leads to better resolution. However, more MISR signatures are needed with smaller $L$; as a result, both storage and diagnosis times are increased. On the other hand, for a given interval length $L$, better resolution can be obtained with smaller detection probability $\alpha$. An appropriate value of $L$ should be, therefore, chosen to balance resolution with storage cost and diagnosis time. As can be seen in the figure, this interval method is suitable only for the faults with relatively small detection probability, i.e., hard-to-detect faults. Faults with high detection probability, i.e., easy-to-detect faults, will cause a large number of intervals to fail and lead to very low diagnostic resolution. Hence, easy-to-detect faults should be handled a priori, e.g., in 


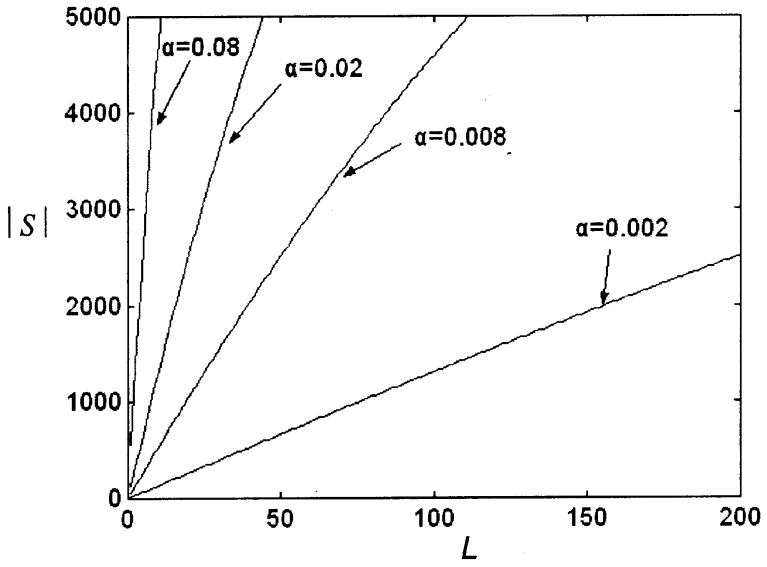

Fig. 5. Size of failing candidate failing vector set $|S|$ versus interval length $L$ $(r=L / 3)$.

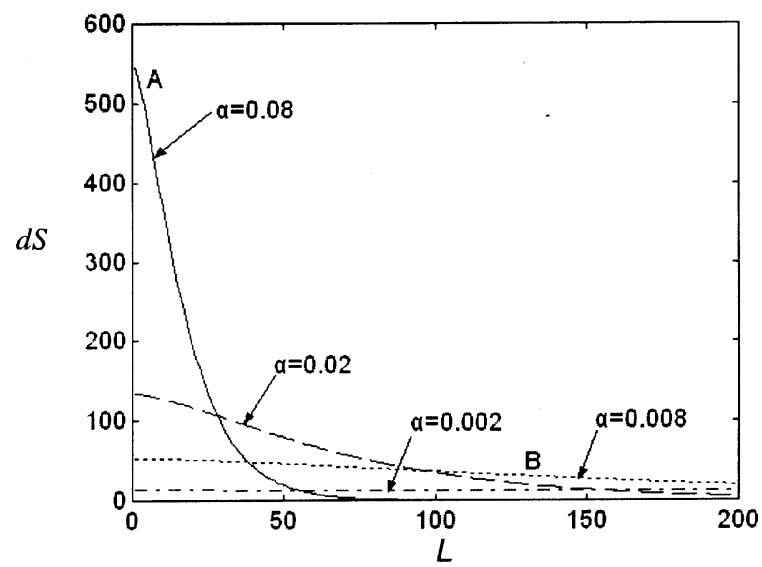

Fig. 6. Rate of increase $d S$ of size of the candidate failing vector set $|S|$ versus interval length $L(r=L / 3)$.

a preprocessing step using a small number of patterns and a very small interval length.

If the failing vector set determined for a specific interval length $L$ is not satisfactory in either resolution or diagnosis time, a different value of $L$ can be chosen and the BIST sessions run again. This procedure can be made more efficient by investigating the rate of increase $d S$ of the candidate set size $|S|$ with $L$, which is obtained by calculating the derivative of $|S|$ with respect to $L$. For example, if $r=L / 3$, we differentiate $|S|$ with respect to $L$ in (1) and get

$$
d S=\frac{\partial|S|}{\partial L}=\frac{N}{2}\left(2 \frac{\partial p}{\partial L}-\frac{\partial p_{1}}{\partial L}+p \frac{\partial p_{1}}{\partial L}+p_{1} \frac{\partial p_{1}}{\partial L}\right) .
$$

Fig. 6 plots $d S$ versus interval length $L$. If better resolution is preferred, $L$ should be chosen near the peak (area $A$ ) of the $d S$ curve such that as $L$ varies, $|S|$ decreases at the highest rate. On the other hand, if shorter diagnosis time is required, $L$ should be chosen near area B such that the variation of $L$ will not result in sharp deterioration in resolution.

Note that for the extreme case of $r=L / 2$, every vector appears in exactly two intervals (except the $L / 2$ vectors in the first interval and the $L / 2$ vectors in the last interval). If the boundary case is omitted, a failing vector always leads to two consecutive failing intervals. This observation can be used to prune $S$

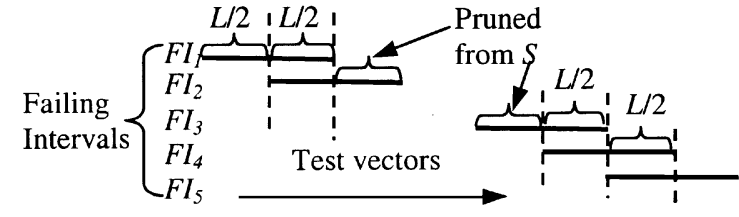

Fig. 7. Pruning paradigm on failing intervals for $r=L / 2$.

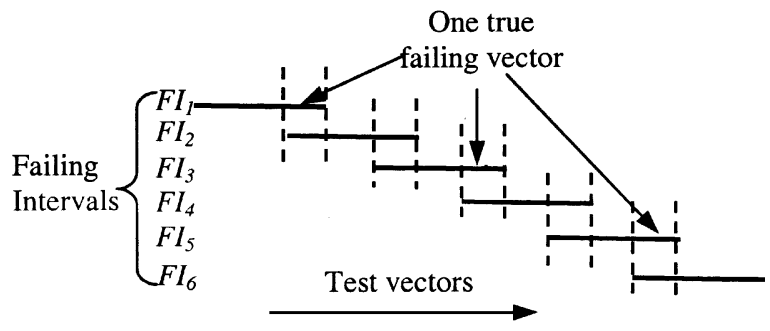

Fig. 8. Worst case of the diagnosis based on pass/fail status of intervals yielding the upper bound $|S|_{\text {up }}$.

as follows. Let $F I_{1}, F I_{2}, \ldots, F I_{m}$ be the sequence of failing intervals where, for any $j>i$, vectors in $F I_{i}$ are applied before those in $F I_{j}$. The candidate failing vector set can be obtained as

$$
\begin{aligned}
S=F I_{1} \cup\left(F I_{1} \cap F I_{2}\right) \cup\left(F I_{2} \cap F I_{3}\right) \\
\ldots \cup\left(F I_{m-1} \cap F I_{m}\right) \cup F I_{m} .
\end{aligned}
$$

This assumes that $F I_{1}$ is the first and $F I_{m}$ is the last interval applied to the CUT. If this is not the case, they each contribute only $L / 2$ vectors to $S$ and the above equation for $S$ must be slightly modified. Fig. 7 illustrates this pruning procedure for $m=5$, where $|S|=L+L+L / 2=5 L / 2$.

Next, we note that if a hard-to-detect fault is detected by only one interval, $|S|=L-r$. In general, if the number of true failing vectors is $f(f \geq 1)$, an upper bound on $|S|$ is given by

$$
|S|_{\text {up }}=2 f(L-r)+r .
$$

This bound is reached if all the failing intervals are consecutive and each failing vector occurs only in overlap sections in every other interval as shown in the example depicted in Fig. 8, where three failing vectors cause six failing intervals and $|S|_{\text {up }}=6 L-$ $5 r$. This is virtually the worst case of using the above pruning procedure based on pass/fail status of intervals. Similarly, given the number of failing intervals $F(F \geq 1)$, a lower bound on $|S|$ is given by

$$
|S|_{\text {low }}=F(L-2 r) \text {. }
$$

This bound is reached if the failing intervals and fault-free intervals are interleaved and the failing vectors occurs only in nonoverlapping sections as in the example in Fig. 9, where two out of the five intervals are failing intervals and $|S|_{\text {low }}=2 L-$ $4 r$. This is the best case of using the above pruning procedure. The knowledge of these two bounds is important for evaluating the performance of the pruning procedure with different distributions of failing intervals.

Finally, we present a graph-theoretic model that can be used to further analyze the interval-based diagnosis approach. In particular, it can be used to determine a lower bound $L B$ on the 


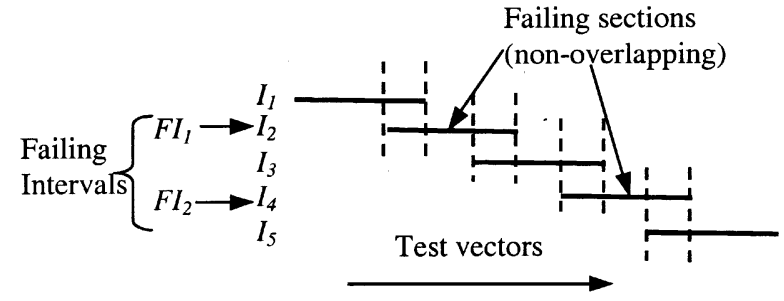

Fig. 9. Best case of diagnosis based on pass/fail status of intervals yielding the lower bound $|S|_{\text {low }}$.

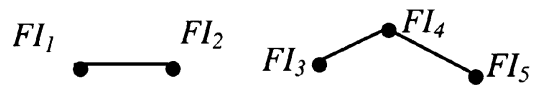

Fig. 10. Graph model for the failing vectors in Fig. 7.

number of true failing vectors. Consider an undirected graph $G$ in which every failing interval corresponds to a vertex, and there exists an edge between two vertices if and only if the corresponding intervals overlap. Let the total number of components in $G$ be $t$ and let $v_{i}$ be the number of vortices in the $i$ th component of $G, 1 \leq i \leq t$. It is clear from the definition of $G$ that every component in it is simply a path. A lower bound is given by

$$
L B=\sum_{i=1}^{t}\left\lfloor\frac{v_{i}+1}{2}\right\rfloor
$$

Consider the failing vectors in Fig. 7 as illustrated in Fig. 10, this corresponds to $t=2, v_{1}=2$, and $v_{2}=3$, hence, $L B=3$. The lower bound on the number of true failing vectors provides an important baseline for evaluating procedures to identify candidate failing vectors.

\section{REducing the Number of CANDidAte FAILING VeCtors}

The pruning procedure introduced in Section III is simply based on the pass/fail status of intervals. However, this approach is often inefficient since only the pass/fail status information is utilized and the advantage of interval overlap is not fully exploited. The inefficiency is easily highlighted in the extreme case of $r=L / 2$. In terms of storage requirement, this is the same as using nonoverlapping intervals with interval length $L / 2$ (the number of signatures is $2 N / L$ in both cases). It can be easily seen that the use of overlapping intervals does not increase the diagnostic resolution in any way. In order to justify the use of overlapping intervals, it is necessary to show that the overlapping scheme always leads to a smaller candidate set than the nonoverlapping scheme based on test windows [18]. We consider next the following two approaches for using intervals of tet vectors:

Approach 1 (OVERLAPPING): overlapping intervals with length $L$ and overlap $r(r<L / 2)$;

Approach 2 (NONOVERLAPPING): nonoverlapping intervals with length $L-r$.

The two methods require the same number of BIST sessions and signatures, hence, their diagnosis overheads are roughly the same. An effective diagnosis scheme based on OVERLAPPING should yield higher resolution (smaller $|S|$ ) than NONOVER$L A P P I N G$ without requiring additional information.

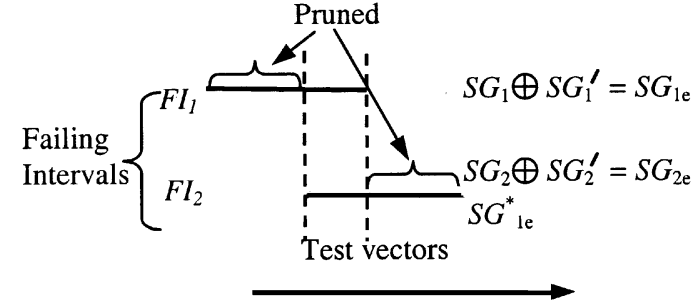

Fig. 11. Pruning fault-free nonoverlapping sections using the principle of superposition.

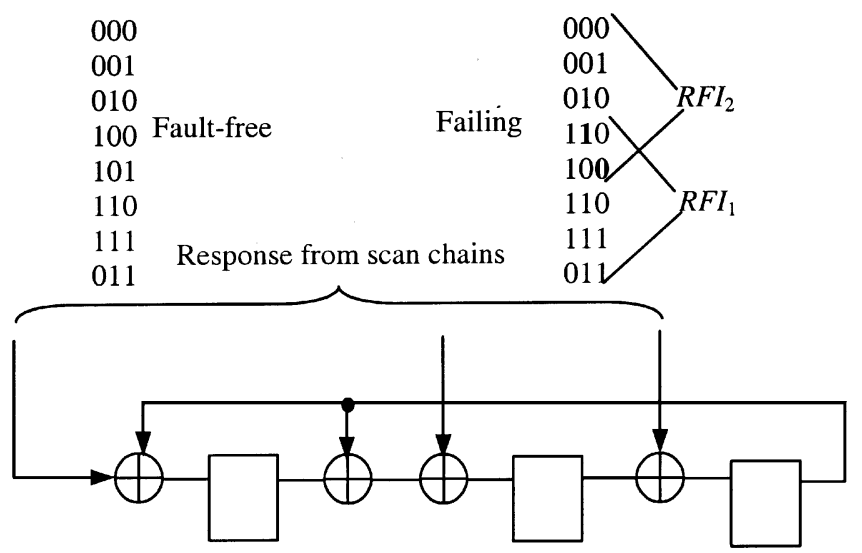

Fig. 12. Pruning example for a MISR with primitive polynomial $l+x+x^{2}$.

From Fig. 8, we observe that failing vector(s) lying in an overlap section can lead to two consecutive failing intervals, even if the nonoverlapping parts of the two intervals are faultfree. A large number of fault-free vectors are unnecessarily included in $S$ due to the failing vector(s) in the overlap section. Moreover, it is easy to see that as interval length decreases, this scenario becomes increasingly likely, which deteriorates diagnostic resolution.

We now present a pruning procedure based on the principle of superposition [6], [20], [22] to solve this problem. In Fig. 11, we show a test sequence with only two failing intervals $F I_{1}$ and $\mathrm{FI}_{2}$ that share a failing overlap section. The two nonoverlapping sections are fault-free. If we apply only the simple pass/fail analysis presented in Section III, the resulting candidate set contains $2 L-r$ vectors since both intervals are deemed as failing. In the following discussion, we assume that the hardware sketched in Fig. 3 is used.

We denote the fault-free signatures for $F I_{1}$ and $F I_{2}$ as $S G_{1}$ and $S G_{2}$, and the corresponding faulty signatures as $S G_{1}^{\prime}$ and $S G_{2}^{\prime}$, respectively. From the principle of superposition, it is well known that $S G_{1} \oplus S G_{1}^{\prime}=S G_{1 e}$ and $S G_{2} \oplus S G_{2}^{\prime}=S G_{2 e}$, where $S G_{1 e}$ and $S G_{2 e}$ are known as error signatures. Now we initialize the MISR, in this case $\mathrm{MISR}_{2}$, with the error signature $S G_{1 e}$ and run it in autonomous mode (inputs set to zero) for as many cycles as needed by $L-r$ scan vectors, and we denote the resulting signature as $S G_{1 e}^{*}$. Under the principle of superposition, the operation of $\mathrm{MISR}_{2}$ can be deemed as the superposition of two processes running simultaneously. The first process feeds the MISR with only fault-free responses and runs it in normal mode while the second process runs the MISR in autonomous mode, initialized to the error signature $S G_{1 e}$. This 


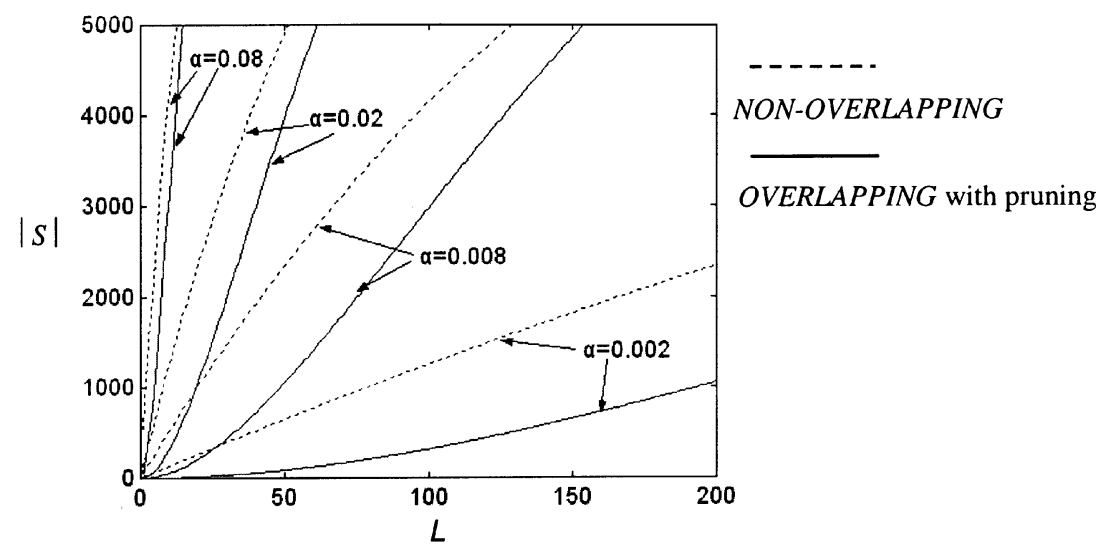

Fig. 13. Size of failing candidate failing vector set $|S|$ versus interval length $L(r=L / 3)$ using NONOVERLAPPING and OVERLAPPING with superposition-based pruning.

can be better explained by the relationship $S G_{1} \oplus S G_{1 e}=S G_{1}^{\prime}$, which indicates that the resulting MISR signature is composed of a fault-free part and an erroneous part. As a result, if $S G_{1 e}^{*}=$ $S G_{2 e}$, we can restrict the failing section to the overlap and the vectors in nonoverlapping sections can be removed from the candidate set. After this step, we reduce the size of the candidate set from $2 L-r$ to $r$, which is a significant improvement on resolution. Note that this pruning step requires no additional information other than fault-free signatures for the intervals. We further note that this procedure is applicable if the nonoverlapping sections are fault free. If the amount of overlap increases, the probability that failing vectors are contained only in overlap sections increases, hence, it is more likely that we can successfully prune the nonoverlapping sections from the candidate set. This explains why OVERLAPPING outperforms NONOVER$L A P P I N G$, where no such pruning is possible.

We illustrate this procedure by an example shown in Fig. 12. We assume that the hardware in Fig. 3 is used and the MISRs have three inputs coming from three scan chains, respectively. The MISRs are constructed using the primitive polynomial $1+$ $x+x^{2}$. Suppose the test sequence for the CUT consists of eight vectors with two intervals of length five $(L=5)$ and overlap two $(r=2)$, and the corresponding responses are eight three-bit vectors fed to the MISR sequentially. (For the sake of simplicity, we assume that the lengths of scan chains are all one.) We denote the two intervals of the test vectors as $F I_{1}$ and $F I_{2}$, and the corresponding two intervals of response stream (input stream to MISR) as $R F I_{1}$ and $R F I_{2}$, respectively. From the fault-free and failing values of $R F I_{1}$ and $R F I_{2}$ shown in Fig. 12, we see that two error bits (shown as bold) occur in the overlapping section of $R F I_{1}$ and $R F I_{2}$, implying that $F I_{1}$ and $F I_{2}$ have a failing overlap and the nonoverlapping sections are fault free. By initializing the MISR to "000" and cycling it with input $R F I_{1}$, we can obtain the various signatures as

$$
S G_{1}=" 111 ", S G_{1}^{\prime}=" 011 ", S G_{1 e}=" 100 "
$$

We do the same calculation for $R F I_{2}$ and the corresponding signatures are

$$
S G_{2}=" 000 ", S G_{2}^{\prime}=" 110 ", S G_{2 e}=" 110 "
$$

Now we reseed the MISR with $S G_{1 e}=$ "100" and advance it for three cycles with all three inputs set to 0 . The resulting signature is

$$
S G_{1 e}^{*}=" 110 "=S G_{2 e} .
$$

This result indicates that only the overlapping section is failing and the nonoverlapping sections are fault-free. Hence, we can reduce the candidate failing vector set from eight to two.

The pruning procedure can occasionally cause aliasing, which may lead to the exclusion of some true failing vectors from the set of candidates. For example, if there are failing vectors in the nonoverlapping section, aliasing can lead to $S G_{1 e}^{*}=S G_{2 e}$. The true failing vectors in the nonoverlapping section are then excluded from the candidate failing vector set. However, the likelihood of this type of aliasing is very low since we consider only hard-to-detect faults, which lead to a small number of true failing vectors. In addition, a longer MISR can also decrease the aliasing probability. We never encountered this type of aliasing in our experiments with the ISCAS benchmark circuits.

The analytical results characterizing the size of the failing vector candidate set presented in Section III must be updated to accommodate the above pruning procedure. For the sake of simplicity, we consider only the best case, where a pair of consecutive intervals have a failing overlapping section and fault-free nonoverlapping sections; and the intervals preceding and following this interval pair are both failing intervals. For each of this interval pair, we can reduce as many as $2 L-2 r$ fault-free vectors from the candidate set given by the simple pass/fail analysis (Section III) with a probability of $\left(1-p_{1}\right) p_{2}^{2}$. Using the result in (1), the updated size of candidate set is given by

$$
\begin{aligned}
|S|=k\left[(1-p) p_{1}(L-2 r)+(\right. & \left.-p_{1}+p p_{1}\right)(L-r) \\
& \left.-2\left(1-p_{1}\right) p_{2}^{2}(L-r)\right] .
\end{aligned}
$$

We plot the updated size of $S$ as a function of $L$ (using $r=L / 3$ and $N=10000$ ) and for various values of $\alpha$ in Fig. 13 using OVERLAPPING. In order to make a comparison, we also plot the corresponding result using NONOVERLAPPING in dotted curves, which can be simply obtained by

$$
|S|=k p_{1}(L-r)
$$




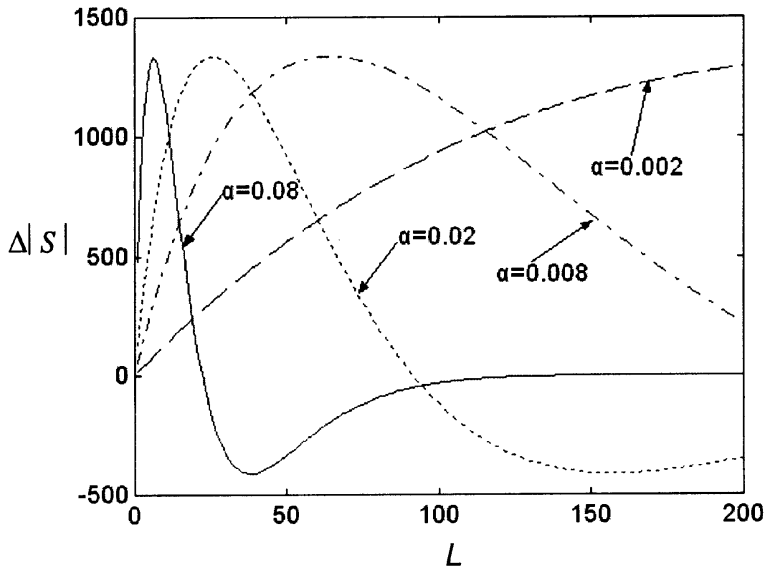

Fig. 14. Improvement of OVERLAPPING over NONOVERLAPPING $\triangle|S|$ versus interval length $L(r=L / 3)$.

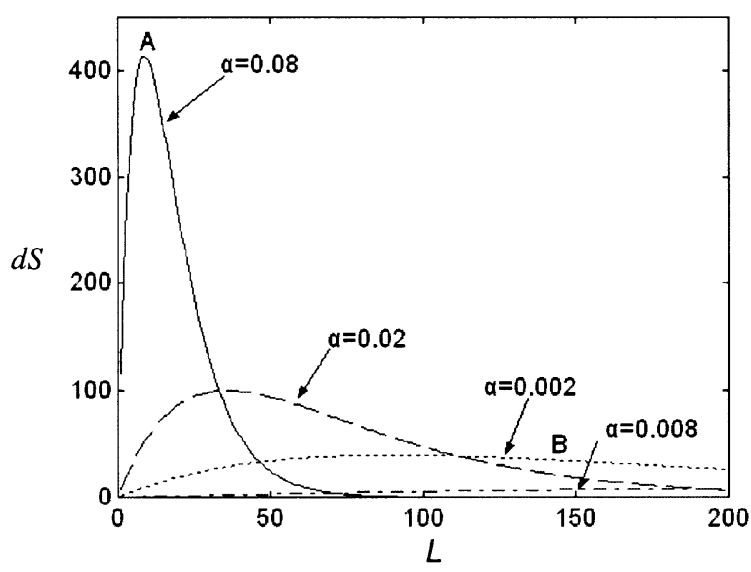

Fig. 15. Rate of increase $d S$ of size of the candidate failing vector set $|S|$ versus interval length $L(r=L / 3)$ with superposition-based pruning.

As expected, a significant reduction in $|S|$ is seen, especially for faults with low detection probability. This justifies our previous argument that the OVERLAPPING interval scheme is more effective for the hard-to-detect faults. This is further illustrated in Fig. 14, where we plot the amount of improvement, $\Delta|S|=$ $|S|_{\text {NONOVERLAPPING }}-|S|_{\text {OVERLAPPING }}$, versus $L$ (using $r=L / 3$ and $N=10000$ ). For easy-to-detect faults, the use of overlap may adversely affect the outcome. This is because the large number of failing vectors can corrupt both the overlapping and nonoverlapping section of an interval, which renders the pruning procedure ineffective. This also occurs when an excessively large $L$ is used. We also plot $d S$ versus interval length $L$ with pruning in Fig. 15. Compared to Fig. 6, the right-shifted peaks indicate that the preferred regions can be attained at larger interval length.

The effectiveness of the OVERLAPPING can also be affected by the amount of overlap $r$. In Fig. 16, we plot $\Delta|S|$ versus $r$ for a given $L=64(N=10000)$, where $r \leq L / 2$. Clearly, for hard-to-detect faults, the OVERLAPPING scheme always outperforms the better results, and $\Delta|S|$ increases monotonously with $r$. However, for easy-to-detect faults, $\Delta|S|$ may decrease as $r$ increases due to the ineffectiveness of the pruning procedure, and $r=L / 2$ denotes the worst case.

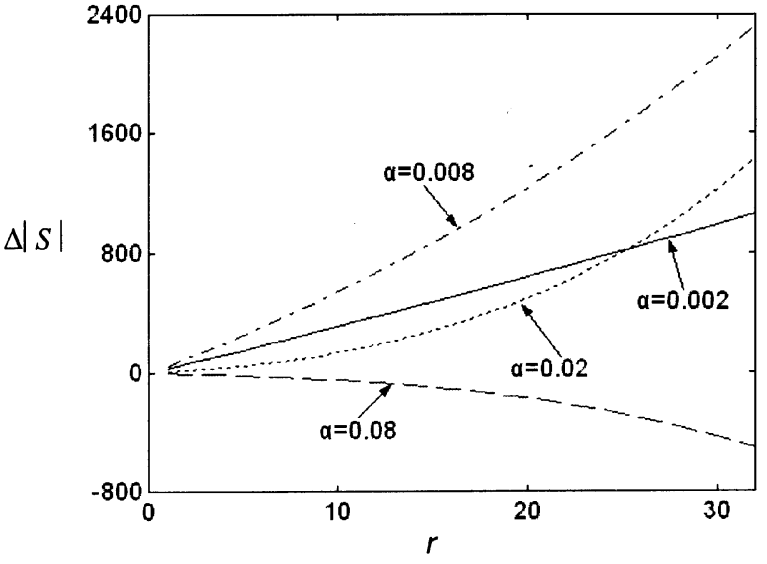

Fig. 16. Improvement of OVERLAPPING over NONOVERLAPPING $\triangle|S|$ versus overlap $r(L=64)$.

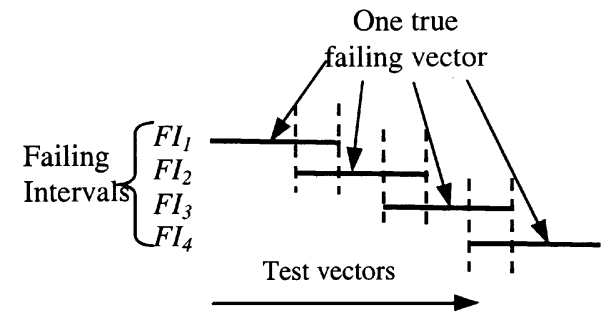

Fig. 17. Worst case of diagnosis with pruning yielding the upper bound $|S|_{\text {up }}$.

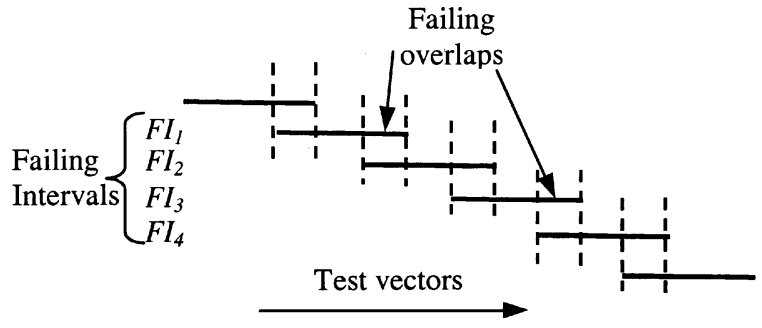

Fig. 18. Best case of the diagnosis with pruning yielding the lower bound $|S|_{\text {low }}$.

Finally, we update the upper bound $|S|_{\text {up }}$ and lower bound $|S|_{\text {low }}$ on the size of candidate failing vector set presented in Section III. Given the number of true failing vectors $f(f \geq 1)$, the new upper bound on $|S|$ is given by

$$
|S|_{\text {up }}=f(L-r)+r .
$$

This bound is reached if all the failing intervals are consecutive and each failing vector appears only in the nonoverlapping section in every interval, as shown in Fig. 17. The four failing vectors in this example cause five failing intervals and $|S|_{\text {up }}=4 L-3 r$. This is the worst case for the pruning procedure since no pruning is possible under this distribution of failing vectors. Similarly, given the number of failing intervals $F(F \geq 1)$, the new lower bound on $|S|$ is given by

$$
|S|_{\text {low }}=\frac{F_{r}}{2} .
$$

This bound is reached if the failing vectors are all in overlapping sections and the distance between two failing overlaps is larger 
TABLE II

EXPERIMENTAL RESULTS ON FAILING VECTOR IDENTIFICATION USING INTERVAL-BASED SCAN-BIST ON 10000 VECTORS

\begin{tabular}{|c|c|c|c|c|c|c|c|c|c|c|c|c|c|}
\hline \multirow[b]{2}{*}{ Circuit } & \multirow{2}{*}{\begin{tabular}{|c|} 
True \\
failing \\
vectors \\
in \\
10,000 \\
BIST \\
vectors
\end{tabular}} & \multirow[b]{2}{*}{$\begin{array}{c}\text { Interval } \\
\text { length } \\
L\end{array}$} & \multicolumn{6}{|c|}{$r=0.33 L$} & \multirow{2}{*}{\begin{tabular}{|c|}
$r=0.4$ \\
$L$ \\
\\
No. of \\
suspect \\
failing \\
vectors \\
$|S|$
\end{tabular}} & \multicolumn{2}{|c|}{$\begin{array}{c}\text { Post-processing I } \\
r=0.33 L \\
\end{array}$} & \multicolumn{2}{|c|}{$\begin{array}{c}\text { Post-processing II } \\
r=0.33 \mathrm{~L}\end{array}$} \\
\hline & & & $\begin{array}{c}\text { No. of } \\
\text { failing } \\
\text { intervals }\end{array}$ & \begin{tabular}{|c|} 
Lower \\
bound \\
$L B$ on \\
no. of \\
true \\
failing \\
vectors \\
\end{tabular} & $\begin{array}{l}\text { Upper } \\
\text { bound } \\
|S|_{\text {up }} \\
\text { on }|S|\end{array}$ & $\left|\begin{array}{l}\text { Lower } \\
\text { bound } \\
|S|_{\text {low }} \\
\text { on }|S|\end{array}\right|$ & $\begin{array}{c}\text { No. of } \\
\text { candidate } \\
\text { failing } \\
\text { vectors }|S| \\
\text { (OVERLAP) }\end{array}$ & $\begin{array}{c}\text { No. of } \\
\text { candidate } \\
\text { failing } \\
\text { vectors }|S| \\
(N O N- \\
\text { OVERLAP) }\end{array}$ & & $\begin{array}{c}\text { No. of } \\
\text { suspect } \\
\text { failing } \\
\text { vectors } \\
|S|\end{array}$ & \begin{tabular}{|c|} 
No. of \\
true \\
failing \\
vectors \\
included \\
in $S$
\end{tabular} & $\begin{array}{c}\text { No. of } \\
\text { suspect } \\
\text { failing } \\
\text { vectors } \\
|S|\end{array}$ & $\begin{array}{c}\text { No. of } \\
\text { true } \\
\text { failing } \\
\text { vectors } \\
\text { included } \\
\text { in } S\end{array}$ \\
\hline \multirow{6}{*}{ s9234 } & 9 & 64 & 11 & 7 & 408 & 115 & 238 & 301 & 188 & 40 & 7 & 40 & 7 \\
\hline & & 32 & 14 & 9 & 200 & 77 & 157 & 189 & 127 & 39 & 7 & 52 & 9 \\
\hline & 36 & 64 & 43 & 28 & 1569 & 451 & 951 & 1333 & 825 & 117 & 30 & 183 & 32 \\
\hline & & 32 & 49 & 31 & 767 & 198 & 388 & 693 & 388 & 156 & 34 & 161 & 35 \\
\hline & 46 & 64 & 56 & 39 & 1999 & 588 & 1168 & 1849 & 1200 & 257 & 39 & 284 & 44 \\
\hline & & 32 & 70 & 43 & 977 & 385 & 590 & 903 & 539 & 191 & 41 & 214 & 45 \\
\hline \multirow{6}{*}{ s13207 } & 22 & 64 & 32 & 20 & 967 & 336 & 558 & 903 & 507 & 106 & 20 & 109 & 21 \\
\hline & & 32 & 32 & 20 & 473 & 176 & 252 & 462 & 275 & 95 & 21 & 103 & 22 \\
\hline & 28 & 64 & 37 & 25 & 1225 & 388 & 712 & 1118 & 566 & 118 & 24 & 124 & 26 \\
\hline & & 32 & 39 & 25 & 599 & 214 & 324 & 567 & 357 & 125 & 27 & 125 & 27 \\
\hline & 92 & 64 & 101 & 63 & 3977 & 1060 & 2308 & 3182 & 2406 & 358 & 63 & 467 & 82 \\
\hline & & 32 & 122 & 76 & 1943 & 671 & 1203 & 1743 & 1077 & 383 & 77 & 431 & 87 \\
\hline \multirow{6}{*}{ s15850 } & 49 & 64 & 53 & 35 & \begin{tabular}{|l|l|}
2128 \\
\end{tabular} & 556 & 1211 & 1806 & 1117 & 233 & 40 & 278 & 47 \\
\hline & & 32 & 68 & 40 & 1040 & 374 & 641 & 903 & 558 & 211 & 42 & 227 & 46 \\
\hline & 68 & 64 & 86 & 48 & 2945 & 903 & 1913 & 2537 & 1886 & 291 & 53 & 341 & 62 \\
\hline & & 32 & 95 & 60 & 1439 & 522 & 806 & 23 & 885 & 279 & 62 & 305 & 67 \\
\hline & 94 & 64 & 104 & 64 & \begin{tabular}{|l|}
4063 \\
\end{tabular} & 1092 & 2395 & 3182 & 2390 & 406 & 65 & 502 & 80 \\
\hline & & 32 & 125 & 76 & 1985 & 687 & 1153 & 1701 & 1093 & 354 & 78 & 410 & 89 \\
\hline \multirow{6}{*}{ s38417 } & 45 & 64 & 52 & 32 & \begin{tabular}{|l|}
1956 \\
\end{tabular} & 546 & 1164 & 1591 & 976 & 186 & 34 & 220 & 39 \\
\hline & & 32 & 57 & 36 & 956 & 313 & 492 & 798 & 508 & 178 & 37 & 201 & 41 \\
\hline & 58 & 64 & 61 & 36 & 2515 & 640 & 1425 & 1849 & 1222 & 207 & 35 & 258 & 43 \\
\hline & & 32 & 70 & 42 & 1229 & 385 & 792 & 1008 & 788 & 216 & 40 & 287 & 54 \\
\hline & 88 & 64 & 112 & 69 & 3805 & 1176 & 2479 & 3421 & 2130 & 420 & 68 & 497 & 81 \\
\hline & & 32 & 121 & 79 & 1859 & 665 & 1067 & 1743 & 992 & 375 & 78 & 411 & 86 \\
\hline \multirow{6}{*}{ s38584 } & 72 & 64 & 94 & 59 & 3117 & 1974 & 1766 & 2795 & 1663 & 352 & 59 & 410 & 67 \\
\hline & & 32 & 98 & 64 & 1523 & 1078 & 825 & 1428 & 760 & 303 & 67 & 329 & 72 \\
\hline & 89 & 64 & 100 & 61 & \begin{tabular}{|l|l|}
3848 \\
\end{tabular} & 1050 & 2395 & 3225 & 2243 & 349 & 60 & 445 & 77 \\
\hline & & 32 & 115 & 74 & 1880 & 632 & 1167 & 1659 & 1120 & 350 & 73 & 409 & 85 \\
\hline & 107 & 64 & 118 & 67 & 4622 & 1239 & 2884 & 3397 & 2412 & 404 & 68 & 519 & 86 \\
\hline & & 32 & 127 & 81 & 2258 & 698 & 1382 & 1890 & 1380 & 399 & 79 & 507 & 100 \\
\hline
\end{tabular}

than $2 L-3 r$, as shown in Fig. 18. Two failing overlaps in this example cause four failing intervals (the first and last intervals are fault-free) and after pruning, $|S|_{\text {low }}=2 r$. This is the best case because all possible fault-free sections are pruned.

We notice that the new upper bound $|S|_{\text {up }}$ is much smaller than the upper bound in (3), and the new $|S|_{\text {low }}$ is larger than the lower bound in (4) if $r>0.4 L$. This suggests that the pruning procedure can potentially provide a smaller range on the theoretically-estimated size of candidate set.

\section{EXPERIMENTAL RESULTS}

In this section, we present simulation results for the larger ISCAS' 89 benchmark circuits. We inject a single random fault in each circuit and use a BIST test sequence of 10000 patterns in the first set of experiments, and 100000 patterns in the second set of experiments. The test patterns are randomly generated and fault simulation is performed using the FSIM program [16]. (The results for s35932 are not included since the random-testable faults in this circuit are easily detected.) Although we limit our experiments to the single stuck-at faults, the proposed interval-based diagnosis can be easily used for other fault models. Our primary objective is to demonstrate the advantage of the OVERLAPPING scheme over the NONOVER$L A P P I N G$ scheme. As discussed in Section IV, the superposition-based pruning technique is not suitable for $r=L / 2$, so we use an overlap of less than $L / 2$ here. In all the experiments, we take the number of sample vectors $N_{i}=N / 100$ to determine $L$ by evaluating $\lfloor 1 / 2 \alpha\rfloor$ in the preprocessing step.

The first set of results is presented in Table II. While we validated our approach for a large number of faults, we report results here only for three faults with different values of $a$ for each circuit. The selected faults have relatively low detection probability. The number of true failing vectors (out of the 10000 applied to the CUT) is shown in Column 2. Column 3 lists various values of interval length $L$. Since for most faults the $L$ values given by the preprocessing step are within a small range around 100, we take $L=64$ for the sake of easy implementation, and then decrease it to 32 to examine the increase resolution. Columns 4-9 present results of failing vector identification using the pruning procedure introduced in Section IV for overlap $r=L / 3$. Column 4 lists the number of failing intervals and Column 5 shows the lower bound on the number of true failing vectors $L B$ attained from the graph model by using the failing interval information. Note that the value of $L B$ is in all cases close to the number of true failing vectors, hence, the former can serve as a good estimate for the latter. Columns 6 and 7 list the upper bound $|S|_{\text {up }}$ and lower bound $|S|_{\text {low }}$, respectively, and Column 8 shows the resulting size of the suspect failing vector set, i.e., the result of OVERLAPPING. In order to make a comparison with the NONOVERLAPPING, we list the result obtained by NONOVERLAPPING with interval 
TABLE III

EXPERIMENTAL RESULTS ON FAILING VeCTOR IDENTIFICATION USING INTERVAL-BASED SCAN-BIST ON 100000 VeCtORS

\begin{tabular}{|c|c|c|c|c|c|c|c|c|}
\hline \multirow[b]{2}{*}{ Circuit } & \multirow[b]{2}{*}{$\begin{array}{c}\text { True } \\
\text { failing } \\
\text { vectors } \\
\text { in } \\
100,000 \\
\text { BIST } \\
\text { vectors } \\
\end{array}$} & \multicolumn{6}{|c|}{$r=0.33 L$} & \multirow[b]{2}{*}{$\begin{array}{c}r=0.4 \mathrm{~L} \\
\\
\text { No. of } \\
\text { suspect } \\
\text { failing } \\
\text { vectors } \\
|S|\end{array}$} \\
\hline & & $\begin{array}{c}\text { No. of } \\
\text { failing } \\
\text { intervals }\end{array}$ & $\begin{array}{c}\text { Lower } \\
\text { bound } L B \\
\text { on no. of } \\
\text { true } \\
\text { failing } \\
\text { vectors }\end{array}$ & $\begin{array}{l}\text { Upper } \\
\text { bound } \\
|S|_{\text {up }} \text { on } \\
|S|\end{array}$ & $\begin{array}{c}\text { Lower } \\
\text { bound } \\
|S|_{\text {low }} \text { on } \\
|S|\end{array}$ & $\begin{array}{c}\text { No. of } \\
\text { candidate } \\
\text { failing } \\
\text { vectors }|S| \\
(O V E R L A P)\end{array}$ & $\begin{array}{c}\text { No. of } \\
\text { candidate } \\
\text { failing } \\
\text { vectors }|S| \\
(N O N- \\
\text { OVERLAP })\end{array}$ & \\
\hline \multirow{3}{*}{ s9234 } & 16 & 25 & 15 & 1403 & 537 & 640 & 1275 & 640 \\
\hline & 28 & 43 & 27 & 2423 & 924 & 1234 & 2295 & 1152 \\
\hline & 51 & 77 & 50 & 4378 & 1655 & 2211 & 4335 & 2360 \\
\hline \multirow{3}{*}{ s13207 } & 14 & 20 & 13 & 1233 & 430 & 637 & 1190 & 609 \\
\hline & 25 & 35 & 24 & 2168 & 752 & 1103 & 2125 & 1112 \\
\hline & 43 & 62 & 41 & 3698 & 1333 & 2038 & 3655 & 1827 \\
\hline \multirow{3}{*}{$\mathrm{s} 15850$} & 9 & 14 & 9 & 808 & 301 & 386 & 765 & 356 \\
\hline & 17 & 27 & 17 & 1488 & 580 & 858 & 1445 & 844 \\
\hline & 50 & 75 & 47 & 4293 & 1612 & 2276 & 4165 & 2312 \\
\hline \multirow{3}{*}{ s38417 } & 18 & 27 & 18 & 1573 & 580 & 765 & 1530 & 793 \\
\hline & 27 & 40 & 27 & 2338 & 860 & 1147 & 2295 & 1127 \\
\hline & 38 & 57 & 36 & 3273 & 1225 & 1871 & 3145 & 1846 \\
\hline \multirow{3}{*}{ s38584 } & 10 & 13 & 10 & 893 & 279 & 423 & 850 & 380 \\
\hline & 21 & 32 & 21 & 1828 & 688 & 893 & 1785 & 868 \\
\hline & 42 & 65 & 40 & 3613 & 1397 & 1873 & 3570 & 1700 \\
\hline
\end{tabular}

length $2 L / 3$ and $r=0$ in Column 9. We notice that the two bounds provide important baselines on the size of candidate failing vector set, and the proposed OVERLAPPING scheme yields a much smaller candidate set than the NONOVERLAP$P I N G$ scheme. This clearly justifies the use of overlap. In Column 10, we increase the amount of overlap to $r=0.4 L$, and as expected, in most cases $|S|$ decreases. (In some cases, $|S|$ increases slightly because the distribution of failing vectors changes, hence, some fault-free sections become faulty and cannot be pruned.) Note that all the true failing vectors out of the 10000 BIST patterns are included in the candidate set. This feature can greatly facilitate the hardware diagnosis procedures that are employed during failure analysis.

If the resulting candidate set obtained, thus far, is still not satisfactory, we apply a pruning-based postprocessing step to further reduce the number of candidates. The methods used here are derived from binary search [21], [23], i.e., failing intervals are partitioned into two equal-sized subsections and test vectors in subsections (branches of the binary tree) are reapplied to determine the pass/fail status of a subsection. The binary partition continues recursively on each failing branch until either a predefined searching depth is reached, or all failing vectors are identified. Since this step is performed after the main step (the OVERLAPPING scheme), we only need to load the fault-free signatures for the failing intervals and reapply the vectors in the failing intervals. If the main step produces $k_{f}$ failing intervals, then a binary search with search depth $d$ needs only the $2^{d} k_{f}$ signatures for the branches on the lowest level of the tree (leaf nodes) because other fault free signatures (for branches on higher levels) can be generated using these $2^{d} k_{f}$ signatures on-the-fly using the principle of superposition [22]. However, the reloading of selected signatures can be done only after the main step and it increases diagnostic cost. In addition, the reapplication of test vectors leads to additional test application time. Therefore, postprocessing is proposed here only as an optional step with a small value of the search depth $d$. We consider two pruning procedures, which are motivated by our choice of interval length-every interval is expected to contain only a few failing vectors. These procedures are different from the standard binary search algorithm in that they do not search all the failing branches. This can reduce the amount of additional test application time with a small amount of information lost on true failing vectors.

In the first pruning procedure I, we assume that a failing interval contains exactly one failing vector. We then use a simple binary search procedure with a predefined searching depth $d$ ( $d=3$ for $L=64$ and $d=2$ for $L=32$ ) to determine the subset of the failing interval that contains the failing vector. This procedure leads to a significant reduction in the size of $S$. A drawback, however, is that a small number of failing vectors are not included in $S$. Nevertheless, as shown in Columns 11 and 12 of Table II, this number is relatively small in all cases.

In the second pruning procedure II, we assume that a failing interval contains at most two failing vectors, and each half of an interval at root level can contain at most one failing vector. We now augment Procedure I to continue the search on both branches of the root node, after which we search only one failing branch on each level. Once again, the search is restricted to a predefined depth $d$ ( $d=3$ for $L=64$ and $d=2$ for $L=32$ ). An advantage of II over I is that fewer true failing vectors are dropped from $S$; see Table II, Columns 13 and 14. The drawback is that more test application time is now necessary due to the increased number of failing branches, and the size of suspect candidate set is slightly increased. Alternative postprocessing pruning procedures can also be employed to reduce the candidate set $S$ provided by the interval-based scan-BIST scheme [8].

In Table III, we present another set of result using 100000 pseudorandom vectors on some faults with even smaller detection probability than those in Table II. These faults are chosen from those that cannot be detected by 10000 pseudorandom patterns. These faults are also not detected by a sample of $N_{i}=$ 1000 vectors in the preprocessing step. Therefore, as discussed in Section II, we set $L=L_{0}=128$, assuming that the associated number of signatures does not exceed the storage capacity. We first note that the size of $S$ is nearly two orders of magnitude less than the test length. We also note that as the detection prob- 
TABLE IV

EXPERIMENTAL RESUlTS AVERAGEd OVER a LARgE NUMBER OF FAULTS ON FAILING VeCTOR IDENTIFICATION USING INTERVAL-BASED SCAN-BIST ON 100000 VECTORS $(L=128, d=3)$

\begin{tabular}{|c|c|c|c|c|c|c|c|c|c|c|c|}
\hline \multirow[b]{2}{*}{ Circuit } & \multirow[b]{2}{*}{$\begin{array}{l}\text { No. of } \\
\text { faults }\end{array}$} & \multirow[b]{2}{*}{$\begin{array}{l}\text { Average } \\
\text { no. of } \\
\text { true } \\
\text { failing } \\
\text { vectors } \\
\text { in } \\
100,000 \\
\text { BIST } \\
\text { vectors }\end{array}$} & \multirow[b]{2}{*}{$\begin{array}{c}\text { Average } \\
\text { no. of } \\
\text { candidate } \\
\text { failing } \\
\text { vectors } \\
|S| \\
(N O N- \\
O V E R L A P \\
\text { PING) }\end{array}$} & \multirow[b]{2}{*}{$\begin{array}{c}\text { Average } \\
\text { no. of } \\
\text { candidate } \\
\text { failing } \\
\text { vectors } \\
|S| \\
(O V E R L A P \\
\text { PING) }\end{array}$} & \multicolumn{3}{|c|}{ Post-processing I } & \multicolumn{3}{|c|}{ Post-processing II } & \multirow[b]{2}{*}{$\begin{array}{c}\text { Average } \\
\text { no. of } \\
\text { candidate } \\
\text { failing } \\
\text { vectors } \\
|S| \\
(O V E R L A P \\
P I N G) \\
k_{1}+k_{2}\end{array}$} \\
\hline & & & & & $\begin{array}{c}\text { Average } \\
\text { no. of } \\
\text { candidate } \\
\text { failing } \\
\text { vectors } \\
|S|\end{array}$ & \begin{tabular}{|c|} 
Average \\
no. of \\
true \\
failing \\
vectors \\
included \\
in $S$
\end{tabular} & $\begin{array}{c}\text { Average } \\
\text { additional } \\
\text { test } \\
\text { application } \\
\text { time } \\
\text { required } \\
(\%)\end{array}$ & $\begin{array}{c}\text { Average } \\
\text { no. of } \\
\text { candidate } \\
\text { failing } \\
\text { vectors } \\
|S|\end{array}$ & \begin{tabular}{|c|} 
Average \\
no. of \\
true \\
failing \\
vectors \\
included \\
in $S$
\end{tabular} & \begin{tabular}{|c|} 
Average \\
additional \\
test \\
application \\
time \\
required \\
$(\%)$
\end{tabular} & \\
\hline s9234 & 1372 & 50 & 4058 & 2393 & 509 & 48 & 6.41 & 522 & 49 & 7.5 & 1553 \\
\hline$\$ \mathbf{s 1 3 2 0 7}$ & 1904 & 27 & 2260 & 1278 & 284 & 26 & 3.59 & 290 & 27 & 4.17 & 990 \\
\hline s15850 & 1045 & 51 & 4140 & 2409 & 519 & 48 & 6.55 & 532 & 50 & 7.65 & 1565 \\
\hline s38417 & 2989 & 21 & 1717 & 967 & 215 & 20 & 2.74 & 219 & 20 & 3.2 & 789 \\
\hline s38584 & 2002 & 48 & 3856 & 2289 & 484 & 45 & 6.08 & 499 & 47 & 7.13 & 1519 \\
\hline
\end{tabular}

ability decreases, the value of the lower bound $L B$ comes very close to the number of true failing vectors (in some cases they are identical), and the ratio of the number of failing intervals to the number of true failing vectors is approximately $L /(L-r)$. In addition, the use of OVERLAPPING leads to candidate sets that are nearly $50 \%$ smaller in size than the candidate size obtained using NONOVERLAPPING. Again, this result justifies the use of interval overlap for hard-to-detect faults.

A direct comparison with related prior work [13] is difficult since the goal of [13] was to obtain a small yet incomplete set of true failing vectors, a large number of true failing vectors are lost. Here, the primary objective is to retain all failing vectors in the candidate set. A secondary objective is to make the candidate set as small as possible. Therefore, it is difficult to conduct a fair comparison between the two methods.

So far, we have presented results on some randomly injected single faults. We now evaluate the performance of the proposed method over a large number of hard-to-detect faults, and thereby derive some statistical insights. In Table IV, we present results on larger ISCAS' 89 benchmarks for a large number of faults and 100000 pseudorandom vectors. These faults are not detected by a sample of $1000\left(N_{i}=1000\right)$ pseudorandom patterns so we set $L=L_{0}=128$. In addition, we set $r=L / 3$. Each column of this table provides an average measure over all the faults, e.g.,

Average number of candidate failing vectors $|S|$

$$
=\frac{\sum_{\text {faults } f} \text { number of candidate failing vectors }|S| \text { for } f}{\text { number of faults }} .
$$

The columns in Table IV are similar to those in Tables I and II except for Columns 8, 11, and 12. Columns 8 and 11 list additional test application time due to the reapplication of test vectors in a binary search procedure. This time is expressed as a percentage of the original test application time without postprocessing. We notice that the results in Table IV are quite similar to those presented in Table III. The average size of $S$ is nearly two orders of magnitude less than the total number of vectors and the average size of $S$ given by the OVERLAPPING scheme is nearly $50 \%$ smaller than those given by the NONOVERLAP$P I N G$ scheme. The use of postprocessing leads to further reduction in the size of $S$; however, the test application time increases
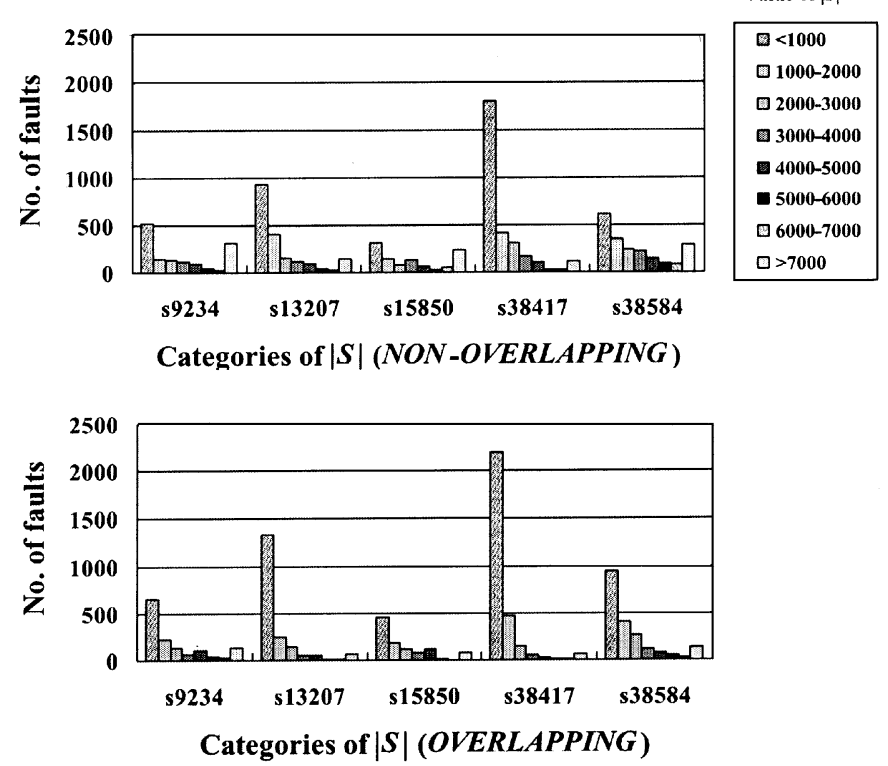

Fig. 19. Number of faults in the various categories of $|S|$ using the NONOVERLAPPING scheme and the OVERLAPPING scheme, respectively.

by up to $7.6 \%$. In Column 12, we show how postprocessing compares to the use of more signatures in a scheme that does not use postprocessing. Suppose the interval-based main step needs $k_{1}$ signatures and postprocessing needs an additional $k_{2}$ signatures. We now simulate the OVERLAPPING scheme (main step) using $k_{1}+k_{2}$ signatures, which indicates a smaller interval length $L$, and list the average size of $S$ in Column 12. The results show that for the same number of signatures, the combination of the interval-based scheme and a postprocessing step can provide better resolution than the interval-based scheme with a smaller interval length. However, we note once again that the postprocessing leads to additional test application time and reloading of signatures, and it can only be applied after the interval-based scheme.

Next, we present another set of experimental results for a large number of faults. Here we use only the superposition-based pruning without postprocessing. For each of the five largest ISCAS' 89 circuits, we considered the same number of faults as listed in Table IV. In Fig. 19, we classify the injected 

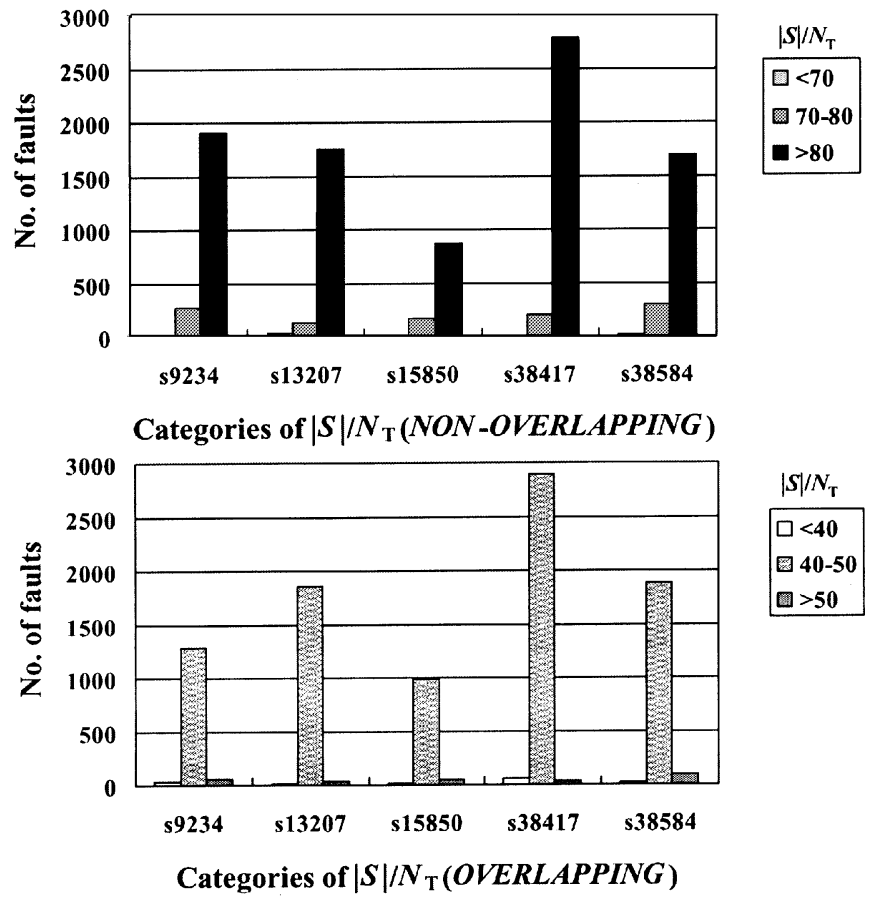

Fig. 20. Number of faults in various categories of $|S| / N T$ using the NONOVERLAPPING scheme and the OVERLAPPING scheme, respectively.

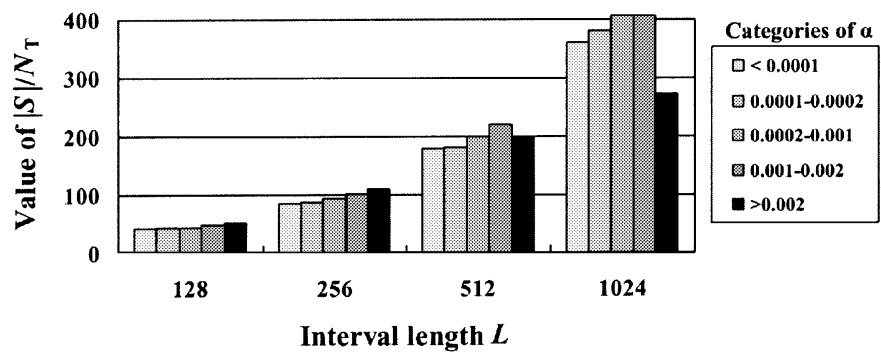

Fig. 21. Value of $|S| / N T$ in various categories of $\alpha$ using the OVERLAPPING scheme with various values of interval length $L$.

faults into different categories on the basis of candidate size $|S|$. It is easy to see that it is desirable for more faults to fall in the categories with smaller $|S|$. Clearly, the OVERLAPPING scheme places many more faults in the categories with smaller values of $|S|$ than the NONOVERLAPPING scheme. Similarly, in Fig. 20, we classify the injected faults into different categories on the basis of the value $|S| / N_{T}$, where $N_{T}$ is the number of true failing vectors. The value of $|S| / N_{T}$ serves as a measure of diagnostic resolution, i.e., a smaller value indicates higher resolution. Again, the OVERLAPPING scheme significantly outperforms the NONOVERLAPPING scheme; over $90 \%$ of the faults in the NONOVERLAPPING scheme have a $|S| / N_{T}$ value larger than 80 , while this value for OVERLAPPING is between $40-50$, which implies a reduction in $|S|$ of about $50 \%$.

Finally, we present results for an experiment in which $\alpha$ is varied and different values of interval length $L$ are used to determine $|S| / N_{T}$. For the sake of simplicity, we consider only one of the largest ISCAS' 89 circuits, namely s38584, and the same faults as listed in Table IV. In Fig. 21, we classify the injected faults into different categories on the basis of $\alpha$, and we present the value of $|S| / N_{T}$ for various values of interval length $L$ using superposition-based pruning without postprocessing. The values of $|S| / N_{T}$ are averaged over all the faults in the corresponding categories. Since $\alpha$ cannot be determined using a sample of $N_{i}=1000$ vectors, we set $L$ to the predefined value $L_{0}$, and $\alpha$ is obtained from $N_{T} / N$ as a posteriori probability. As expected, when $L$ is sufficiently small $(L<512)$, $|S| / N_{T}$ increases monotonically with $\alpha$, but $L$ is the dominant factor influencing the value of $|S| / N_{T}$. However, when $L$ becomes larger, $|S| / N_{T}$ does not increase monotonically with $\alpha$. This set of results provides further justification for our claim that the interval-based technique with superposition-based pruning is suitable for hard-to-detect faults.

\section{CONCLUSION}

We have presented a new scan-BIST diagnosis technique for the identification of failing vectors. This technique is based on the concept of overlapping intervals of test vectors within a BIST sequence. The scan-BIST architecture allows us to collect interval signatures without the need for multiple sessions. Based on the principle of superposition, we have proposed a pruning method by which the candidate failing vector set can be significantly reduced without any additional information. We have presented upper and lower bounds on the size of candidate failing vector set and lower bound on the number of true failing vectors. These bounds can be used as important parameters to evaluate the performance of the diagnosis procedure. Experimental results for the ISCAS' 89 benchmark circuits show that the interval-based scan-BIST method with pruning procedure can reduce the size of the candidate failing vectors set $S$ significantly, and all failing vectors are retained in $S$. Compared to a scheme based on nonoverlapping intervals, the use of overlapping intervals leads to much higher diagnostic resolution with nearly the same overhead. Finally, we have presented results for two simple pruning methods that lead to much smaller candidate sets that include nearly all the true failing vectors. The reduction in the size of the candidate set is accompanied by additional storage requirements and processing time.

\section{REFERENCES}

[1] M. L. Bushnell and V. D. Agrawal, Essentials of Electronic Testing. Norwell, MA: Kluwer, 2000.

[2] P. H. Bardell and W. H. McAnney, "Self-Testing of multichip logic modules," in Proc. Int. Test Conf., 1982, pp. 200-204.

[3] P. Song, F. Motika, D. R. Knebel, R. F. Rizzolo, and M. P. Kusko, "S/390 G5 CMOS microprocessor diagnostics," in Proc. Int. Test Conf., 1999, pp. 1073-1082.

[4] Y. Wu and S. Adham, "BIST fault diagnosis in scan-based VLSI environment," in Proc. Int. Test Conf., 1996, pp. 48-57.

[5] J. Rajski and J. Tyszer, "Fault diagnosis in scan-based BIST," in Proc. Int. Test Conf., 1997, pp. 894-902.

[6] I. Bayraktaroglu and A. Orailoglu, "Improved fault diagnosis in scanbased BIST via superposition," in Proc. Design Automation Conf., June 2000 , pp. 55-58.

[7] - "Diagnosis for scan-based BIST: Reaching deep into the signatures," in Proc. Design Automation Test Eur., Mar. 2001, pp. 102-109.

[8] W. H. McAnney and J. Savir, "There is information in faulty signatures," in Proc. Int. Test Conf., 1987, pp. 630-636.

[9] J. Savir and W. H. McAnney, "Identification of failing tests with cycling registers," in Proc. Int. Test Conf., 1988, pp. 322-328.

[10] T. R. Damarla, C. E. Stroud, and A. Sathaye, "Multiple error detection and identification via signature analysis," J. Electron. Testing: Theory Applicat., vol. 7, pp. 193-207, 1995. 
[11] M. G. Karpovsky and S. M. Chaudhry, "Design of self-diagnosing boards by multiple signature analysis," IEEE Trans. Comput., vol. 42, pp. 1035-1044, Sept. 1993.

[12] R. C. Aitken and V. K. Agarwal, "A diagnosis method using pseudorandom vectors without intermediate signatures," in Proc. Int. Conf. Computer-Aided Design, 1989, pp. 574-580.

[13] J. Ghosh-Dastidar, D. Das, and N. A. Touba, "Fault diagnosis in scanbased BIST using both time and space information," in Proc. Int. Test Conf., 1999, pp. 95-102.

[14] B. Chess and T. Larrabee, "Creating small fault dictionaries," IEEE Trans. Computer-Aided Design, vol. 18, pp. 346-356, Mar. 1999.

[15] I. Pomeranz and S. M. Reddy, "On dictionary-based fault location in digital logic circuits," IEEE Trans. Comput., vol. 46, pp. 48-59, Jan. 1997.

[16] H. K. Lee and D. S. Ha, "An efficient forward fault simulation algorithm based on the parallel pattern single fault propagation," in Proc. Int. Test Conf., 1991, pp. 946-955.

[17] Y. Wu and A. Ivanov, "Single-reference multiple intermediate signature (SREMIS) analysis for BIST," IEEE Trans. Comput., vol. 44, pp. 817-825, June 1995.

[18] J. Savir, "Salvaging test windows in BIST diagnostics," in Proc. VLSI Testing Symp., 1997, pp. 416-425.

[19] C. Liu, K. Chakrabarty, and M. Goessel, "An interval-based diagnosis scheme for identifying failing vectors in a scan-BIST environment," in Proc. Design Automation Test Eur. Conf., 2002, pp. 382-386.

[20] J. C. Chan and J. A. Abraham, "A study of faulty signatures using a matrix formulation," in Proc. Int. Test Conf., 1990, pp. 553-561.

[21] T. H. Cormen, C. E. Leiserson, R. L. Rivest, and C. Stein, Introduction to Algorithms. Cambridge, MA: MIT Press, 2001.

[22] P. H. Bardell, W. H. McAnney, and J. Savir, Built-In Test for VLSI: Pseudorandom Techniques. New York: Wiley, 1987.

[23] J. Ghosh-Dastidar and N. A. Touba, "A rapid and scalable diagnosis scheme for BIST environment with a large number of scan chains," in Proc. VLSI Test Symp., 2000, pp. 79-85.

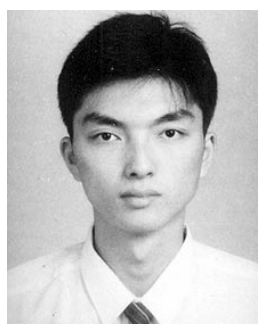

Chunsheng Liu (S'00) received the B.S. and M.S. degrees in electronic engineering from Tsinghua University, Beijing, China, in 1997 and 2000, respectively. He is currently pursuing the Ph.D. degree in the Department of Electrical and Computer Engineering, Duke University, Durham, NC.

His research interests include VLSI design, testing, and computer-aided design tools. $\mathrm{He}$ is currently working in the areas of fault diagnosis, system-on-a-chip (SOC) testing and scan-based BIST.

Mr. Liu is a member of the Association for Computing Machinery (ACM) Special Interest Group on Design Automation.

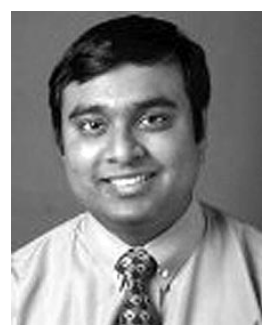

Krishnendu Chakrabarty (S'91-M'95-SM'00) received the B.Tech. degree from the Indian Institute of Technology, Kharagpur, India, in 1990, and the M.S.E. and Ph.D. degrees from the University of Michigan, Ann Arbor, in 1992 and 1995, respectively, all in computer science and engineering.

$\mathrm{He}$ is currently an Associate Professor of Electrical and Computer Engineering at Duke University, Durham, NC. From 2000 to 2002, he was also a Mercator Visiting Professor at the University of Potsdam, Potsdam, Germany. His current research projects (supported by the National Science Foundation, and the Defense Advanced Research Projects Agency, the Office of Naval Research, the Army Research Office, and industrial sponsors) are in system-on-a-chip test, embedded real-time operating systems, distributed sensor networks and modeling, simulation, and optimization of microelectrofluidic systems. $\mathrm{He}$ is a coauthor of two books: Microelectrofluidic Systems: Modeling and Simulation (Boca Raton, FL: CRC, 2002) and Test Resource Partitioning for System-on-a-Chip (Norwell, MA: Kluwer, 2002) and an editor of the book SOC (System-on-a-Chip) Testing for Plug and Play Test Automation (Norwell, MA: Kluwer, 2002). He has published over 120 papers in archival journals and refereed conference proceedings, and holds a U.S. patent in BIST.

Dr. Chakrabarty is a member of ACM and the ACM Special Interest Group on Design Automation, and a member of Sigma Xi. He is a recipient of the National Science Foundation Early Faculty (CAREER) Award, the Office of Naval Research Young Investigator Award and a best paper award at the 2001 Design, Automation, and Test in Europe Conference. He is an Associate Editor of IEEE TRANSACTIONS ON COMPUTER-AIDED DESIGN OF INTEGRATED CIRCUITS AND SYSTEMS, an Editor of the Journal of Electronic Testing: Theory and Applications (JETTA), and the Guest Editor of a special issue of JETTA on system-on-a-chip testing, published in August 2002. He was also a Guest Editor of a special issue of the Journal of the Franklin Institute on distributed sensor networks in 2001. He serves as Vice Chair of Technical Activities of IEEE's Test Technology Technical Council and is a Member of the Program Committees of several IEEE/ACM conferences and workshops. 